University of Wollongong

Research Online

Australian Institute for Innovative Materials -

Papers

Australian Institute for Innovative Materials

$1-1-2015$

The effect of symmetry on resonant and nonresonant photoresponses in a field-effect terahertz detector

J D. Sun

Chinese Academy Of Sciences

H Qin

Chinese Academy Of Sciences

Roger A. Lewis

University of Wollongong, roger@uow.edu.au

Xiaoyu Yang

Chinese Academy Of Sciences, kxy@cnic.cn

Y F. Sun

Suzhou University of Sciences And Technology

See next page for additional authors

Follow this and additional works at: https://ro.uow.edu.au/aiimpapers

Part of the Engineering Commons, and the Physical Sciences and Mathematics Commons

Research Online is the open access institutional repository for the University of Wollongong. For further information contact the UOW Library: research-pubs@uow.edu.au 


\title{
The effect of symmetry on resonant and nonresonant photoresponses in a field- effect terahertz detector
}

\author{
Abstract \\ The effect of the symmetries in the terahertz $(\mathrm{THz})$ field distribution and the field-effect channel on $\mathrm{THz}$ \\ photoresponse is examined. Resonant excitation of cavity plasmon modes and nonresonant self-mixing \\ of $\mathrm{THz}$ waves are demonstrated in a GaN/AIGaN two-dimensional electron gas with symmetrically \\ designed nanogates, antennas, and filters. We found that the self-mixing signal can be effectively \\ suppressed by the symmetric design and the resonant response benefits from the residual asymmetry. \\ The findings suggest that a single detector may provide both high sensitivity from the self-mixing \\ mechanism and spectral resolution from the resonant response by optimizing the degree of geometrical \\ and/or electronic symmetries.

\section{Keywords} \\ terahertz, field, photoresponses, detector, nonresonant, symmetry, resonant, effect \\ Disciplines \\ Engineering | Physical Sciences and Mathematics

\section{Publication Details} \\ Sun, J. D., Qin, H., Lewis, R. A., Yang, X. X., Sun, Y. F., Zhang, Z. P., Li, X. X., Zhang, X. Y., Cai, Y., Wu, D. M., \\ Zhang, B. S. et al (2015). The effect of symmetry on resonant and nonresonant photoresponses in a field- \\ effect terahertz detector. Applied Physics Letters, 106 (3), 031119-1-031119-5.

\section{Authors} \\ J D. Sun, H Qin, Roger A. Lewis, Xiaoyu Yang, Y F. Sun, Zhenpu Zhang, Xianghu Li, Xiaoqin Zhang, Y Cai, D \\ M. Wu, and B S. Zhang
}




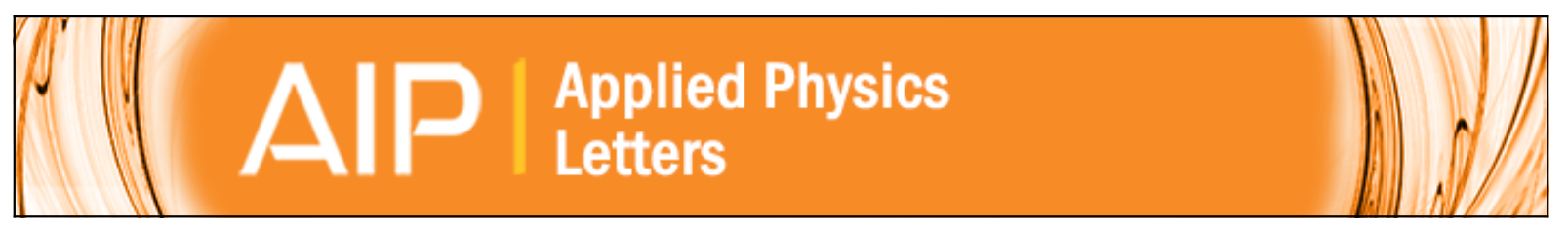

The effect of symmetry on resonant and nonresonant photoresponses in a field-effect terahertz detector

J. D. Sun, H. Qin, R. A. Lewis, X. X. Yang, Y. F. Sun, Z. P. Zhang, X. X. Li, X. Y. Zhang, Y. Cai, D. M. Wu, and B. S. Zhang

Citation: Applied Physics Letters 106, 031119 (2015); doi: 10.1063/1.4906536

View online: http://dx.doi.org/10.1063/1.4906536

View Table of Contents: http://scitation.aip.org/content/aip/journal/apl/106/3?ver=pdfcov

Published by the AIP Publishing

\section{Articles you may be interested in}

Modeling an antenna-coupled graphene field-effect terahertz detector

Appl. Phys. Lett. 103, 173507 (2013); 10.1063/1.4826118

Probing and modelling the localized self-mixing in a GaN/AIGaN field-effect terahertz detector

Appl. Phys. Lett. 100, 173513 (2012); 10.1063/1.4705306

High-responsivity, low-noise, room-temperature, self-mixing terahertz detector realized using floating antennas on a GaN-based field-effect transistor

Appl. Phys. Lett. 100, 013506 (2012); 10.1063/1.3673617

Room temperature GaN/AIGaN self-mixing terahertz detector enhanced by resonant antennas

Appl. Phys. Lett. 98, 252103 (2011); 10.1063/1.3601489

Influence of $\mathrm{AlGaN} / \mathrm{GaN}$ interface polarization fields on the properties of photoconductive detectors J. Appl. Phys. 95, 5925 (2004); 10.1063/1.1699522

High-Voltage Amplifiers

- Voltage Range from $\pm 50 \mathrm{~V}$ to $\pm 60 \mathrm{kV}$

- Current to $25 \mathrm{~A}$

Electrostatic Voltmeters

- Contacting \& Non-contacting

- Sensitive to $1 \mathrm{mV}$

- Measure to $20 \mathrm{kV}$
ENABLING RESEARCH AND

INNOVATION IN DIELECTRICS,

ELECTROSTATICS,

MATERIALS, PLASMAS AND PIEZOS

Rek www.trekinc.com

TREK, INC. 190 Walnut Street, Lockport, NY 14094 USA •Toll Free in USA 1-800-FOR-TREK• (t):716-438-7555 • (f):716-201-1804 • sales@trekinc.com 


\title{
The effect of symmetry on resonant and nonresonant photoresponses in a field-effect terahertz detector
}

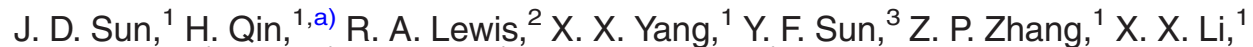 \\ X. Y. Zhang, ${ }^{1}{ }^{\text {Y. Cai, }}{ }^{1}$ D. M. Wu, ${ }^{1}$ and B. S. Zhang ${ }^{1}$ \\ ${ }^{1}$ Key Laboratory of Nanodevices and Applications, Suzhou Institute of Nano-tech and Nano-bionics, \\ Chinese Academy of Sciences, 398 Ruoshui Road, Suzhou, Jiangsu 215123, People's Republic of China \\ ${ }^{2}$ Institute for Superconducting and Electronic Materials, University of Wollongong, Wollongong, \\ New South Wales 2522, Australia \\ ${ }^{3}$ College of Electronic and Information Enging, Suzhou University of Sciences and Technology, Suzhou, \\ Jiangsu 215011, People's Republic of China
}

(Received 16 December 2014; accepted 13 January 2015; published online 23 January 2015)

\begin{abstract}
The effect of the symmetries in the terahertz ( $\mathrm{THz}$ ) field distribution and the field-effect channel on $\mathrm{THz}$ photoresponse is examined. Resonant excitation of cavity plasmon modes and nonresonant self-mixing of $\mathrm{THz}$ waves are demonstrated in a GaN/AlGaN two-dimensional electron gas with symmetrically designed nanogates, antennas, and filters. We found that the self-mixing signal can be effectively suppressed by the symmetric design and the resonant response benefits from the residual asymmetry. The findings suggest that a single detector may provide both high sensitivity from the self-mixing mechanism and spectral resolution from the resonant response by optimizing the degree of geometrical and/or electronic symmetries. (C) 2015 AIP Publishing LLC.

[http://dx.doi.org/10.1063/1.4906536]
\end{abstract}

In the terahertz $(\mathrm{THz})$ portion of the electromagnetic spectrum, many sensing applications, such as security screening, near-field microscopy, and spectroscopy, are being studied and developed. ${ }^{1}$ Sensitive, tunable, and compact semiconductor detectors are one of the key elements for such applications. ${ }^{2}$ In the early 1990s, Dyakonov and Shur theoretically predicted that plasmon waves in the field-effect electron channel can be applied for $\mathrm{THz}$ emission and detection. ${ }^{3,4}$ For detection, specifically, plasmon waves can be excited either resonantly or nonresonantly (self-mixing) upon $\mathrm{THz}$ irradiation. ${ }^{4}$ It is naturally expected that a resonant response would allow for more sensitive detection and also allow for spectral resolution. ${ }^{4-12}$ However, experimental results reported so far show that the resonant response is much weaker than the nonresonant response and the former is usually submerged into the latter. ${ }^{5-7}$ The amplitude of the resonant response is limited by the quality factor $\left(Q=\omega_{p} \tau_{p}\right.$, where $\omega_{p}$ is the plasmon's angular frequency and $\tau_{p}$ is the plasmon's relaxation time) of the plasmon resonance. Understanding the physics in plasmon damping would allow us to control the plasmon cavity with proper boundary conditions and to develop high-performance plasmon-based $\mathrm{THz}$ devices.

In the resonant case $\left(\omega_{p} \tau_{p} \gg 1\right)$, a plasmon wave is excited resonantly at a proper gate voltage and induces an unidirectional photocurrent which has a Lorentzian line shape $^{4-7}$

$$
i_{R}=I_{R} \frac{f_{p}^{2}}{\left(f-f_{p}\right)^{2}+\left(1 / 4 \pi \tau_{p}\right)^{2}}
$$

where prefactor $I_{R}$ is a complex function of the incident $\mathrm{THz}$ power, channel conductance, the geometry, and the boundary

\footnotetext{
${ }^{a)}$ Electronic mail: hqin2007@sinano.ac.cn
}

conditions, $f$ is the incident THz frequency, and $f_{p}$ is the plasmon frequency which, according to Refs. 3 and 4, can be tuned by the gate voltage

$$
f_{p}=\frac{1}{4 L_{e f f}} \sqrt{\frac{e U_{g}}{m^{*}}},
$$

where $L_{\text {eff }}$ is the effective length of the plasmon cavity, and $e$ and $m^{*}$ are the electron charge and effective mass, respectively. In the nonresonant case $\left(\omega_{p} \tau_{p} \ll 1\right)$, the photoresponse comes from self-mixing of the $\mathrm{THz}$ electric field in the electron channel and is effective over a wide range of the electron density, i.e., has a broadband response with the bandwidth determined by the THz antenna. ${ }^{13-19}$ The shortcircuit self-mixing current can be written as ${ }^{18}$

$$
i_{M}=\frac{E_{0}^{2}}{4} \bar{z} \int_{0}^{L} \frac{\mathrm{d} G}{\mathrm{~d} U_{g}} \dot{\xi}_{x} \dot{\xi}_{z} \cos \phi \mathrm{d} x,
$$

where $E_{0}$ is the free-space electric field induced by the incident $\mathrm{THz}$ wave, $G=G(x)$ is the local conductance of the gated channel, $\bar{z}$ is the effective distance between the gate and the channel, $L$ is the length of the channel. $U_{g}=V_{g}$ $-V_{t h}-V_{x}$ is effective gate voltage, $V_{g}, V_{t h}$, and $V_{x}$ are the applied voltage, the threshold voltage, and the local channel potential, respectively. $\dot{\xi}_{x}, \dot{\xi}_{z}$, and $\phi$ are the horizontal and perpendicular $\mathrm{THz}$ field enhancement factors, and the phase difference between the induced fields, respectively. In an antenna coupled field-effect detector, both responses are expected to take place and the total photocurrent can be expressed as

$$
i_{T}=i_{M}+i_{R}
$$

Both self-mixing and resonant responses rely on the asymmetries in the $\mathrm{THz}$ field distribution or the plasmon 
cavity's boundary conditions. For resonant detection, the source/drain ohmic contacts usually serve as the boundaries for the plasmon cavity and also as part of the $\mathrm{THz}$ antennas. The asymmetric boundary conditions for plasmon wave were achieved by biasing the device with a direct current source ${ }^{8-10}$ For self-mixing detection, the asymmetric condition is reached either by using asymmetric antennas or biasing the electron channel with a proper current. ${ }^{13,17}$ Here, we report the effect of symmetry on $\mathrm{THz}$ photoresponse in a $\mathrm{GaN} / \mathrm{AlGaN} \mathrm{THz}$ detector. The detector is based on $\mathrm{GaN} /$ AlGaN two-dimensional electron gas with symmetrically arranged nanogates, antennas, and filters. The three gates form a tunable plasmon cavity with nearly symmetric boundaries. The metallic source/drain contacts are set $1.5 \mu \mathrm{m}$ away from the plasmon cavity to maintain the degree of symmetry. Both self-mixing response and resonant response are observed. By suppressing the self-mixing response, the resonant response is clearly resolved at $77 \mathrm{~K}$. The $\mathrm{THz}$ response as a function of the gate voltage and the sourcedrain bias is mapped for the verification of the symmetric design.

A scanning-electron micrograph of the detector is shown in Fig. 1. Three nanogates are defined as $G_{1}, G_{2}$, and $G_{3}$. A plasmon cavity is formed between gates $\mathrm{G}_{2}$ and $\mathrm{G}_{3}$. Gate $\mathrm{G}_{1}$ applied with voltage $V_{G}$ is used to control the carrier density and thus tune the plasmon frequency. Antennae $\mathrm{A}_{1}$ and $\mathrm{A}_{2}$ are designed to have a resonant frequency at $900 \mathrm{GHz}$ and is connected to gate $G_{1}$ and $G_{2} / G_{3}$, respectively. The meandershaped low pass filters are used to connect the antennas with the corresponding wire-bonding pads. ${ }^{20}$ The gate length is $150 \mathrm{~nm}, 100 \mathrm{~nm}$, and $100 \mathrm{~nm}$ for gates $\mathrm{G}_{1}, \mathrm{G}_{2}$, and $\mathrm{G}_{3}$, respectively. The distance between the inner edges of gate $\mathrm{G}_{2}$ and gate $\mathrm{G}_{3}$ is about $330 \mathrm{~nm}$. The width of the electron channel (mesa) is $W=3 \mu \mathrm{m}$, and the distance between the source and drain contacts is $3.5 \mu \mathrm{m}$. At $77 \mathrm{~K}$, the electron mobility and the electron density are $\mu=1.58 \times 10^{4} \mathrm{~cm}^{2} / \mathrm{Vs}$ and $n_{s}=1.06 \times 10^{13} \mathrm{~cm}^{-2}$, respectively. The effective electron mass is $m^{*}=0.2 m$, where $m$ is the free electron mass. The radiation from a backward wave oscillator (BWO) was modulated by a mechanical chopper at frequency $f_{M}=317 \mathrm{~Hz}$. The short-circuit photocurrent was measured using a current preamplifier and a lock-in amplifier. More experimental details can be found in Ref. 18. The
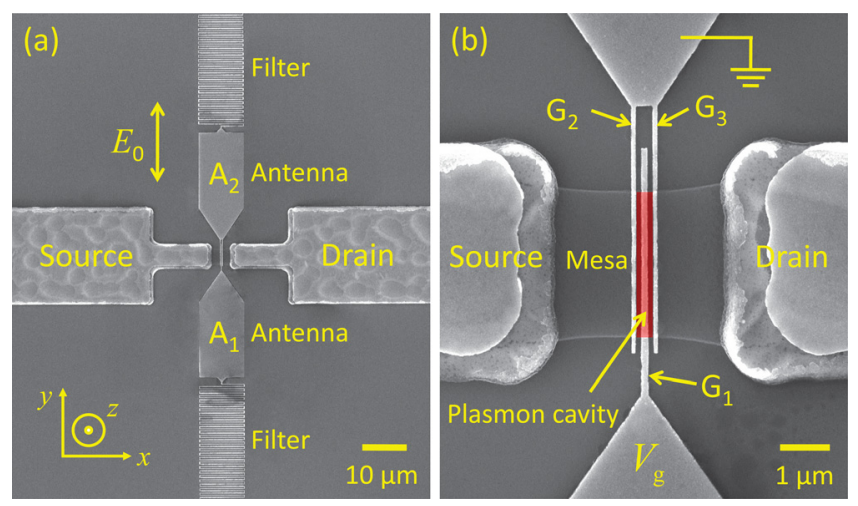

FIG. 1. (a) Scanning-electron micrograph of the field-effect $\mathrm{THz}$ detector. (b) Zoom-in view of the central active region including the plasmon cavity, the nanogates, and the 2DEG channel. photocurrent is maximized when the polarization of the $\mathrm{THz}$ wave (linearly polarized) emitted from the BWO becomes in parallel with the antennas along direction $y$.

As shown in Fig. 2, we present numerical calculations of the $\mathrm{THz}$ electric field and the phase distributions at $900 \mathrm{GHz}$ by a finite-difference time-domain (FDTD) method. To obtain the fine field distribution in the gated 2DEG, the grid constant is set as $5 \mathrm{~nm}$ and $20 \mathrm{~nm}$ in direction $x$ and direction $y$ in FDTD simulations, respectively. The field enhancement factors for the horizontal and perpendicular fields are shown in Figs. 2(a) and 2(b), respectively. The horizontal field is concentrated in the extended areas between gates $G_{1}, G_{2}$, and $G_{3}$, while the perpendicular field is mainly distributed under gate $G_{1}$. The simulation confirms that, unlike the asymmetric detector in Ref. 18, the source and drain sides have the same electric field distribution. The horizontal field changes its phase by $180^{\circ}$ at the center of the three gates, while the perpendicular field changes its phase at the center of the gaps between the gates (Figs. 2(c) and 2(d)). In Fig. 2(e), the simulated mixing factor $\left(\dot{\xi}_{x} \dot{\xi}_{z} \cos \phi\right)$ is plotted to reveal the spatial distribution. Strong mixing symmetrically occurs only at the left and right edges of gate $\mathrm{G}_{1}$. However, the mixing at the source side generates a positive current, opposite to that induced at the drain side. This inversion of polarity comes from a phase flips as shown in Figs. 2(c) and 2(d). Since the device structure is symmetric along the electron channel, the self-mixing response is expected to be suppressed effectively according to Eq. (3).

As shown in Fig. 3(a), the source-drain conductance $G$ and its derivative $\mathrm{d} G / \mathrm{d} V_{g}$ as a function of the gate voltage are characterized at $77 \mathrm{~K}$. The derivative is maximized at $-4.00 \mathrm{~V}$. Fig. 3(b) shows the photocurrent versus $V_{g}$ at different $\mathrm{THz}$ frequencies of $850 \mathrm{GHz}(\square), 861 \mathrm{GHz}(\bigcirc)$, $907 \mathrm{GHz}(\triangle)$, and $940 \mathrm{GHz}(\nabla)$. For the sake of clarity, the curves are shifted in the vertical scale. Two peaks are observed with one of them fixed at $-4.00 \mathrm{~V}$ and the other at a lower gate voltage around $-3 \mathrm{~V}$, depending on the $\mathrm{THz}$ frequency. The frequency-independent response at the same location where the derivative of the conductance is maximal comes from the self-mixing as described by Eq. (3). The frequency-dependent response is induced by the excitation of cavity plasmons and can be well described by Eq. (1). The higher the $\mathrm{THz}$ frequency, the lower the peak gate voltage (i.e., the higher the electron density) will be. When we tune the $\mathrm{THz}$ frequency from $850 \mathrm{GHz}$ to $940 \mathrm{GHz}$, the position of the resonant response shifts from $-3.12 \mathrm{~V}$ to $-2.60 \mathrm{~V}$. Solid curves in Fig. 3(b) are calculated photoresponses based on Eq. (4) and agree well with the experiments. By normalizing the total photoresponse and substracting the self-mixing response, the net resonant responses at different $\mathrm{THz}$ frequencies are shown in Fig. 4(a). The resonant frequency as a function of the gate voltage fits well with Eq. (2), as shown in Fig. 4(b). In the fitting, the size of the plasmon cavity is chosen to be $L_{\text {eff }}=330 \mathrm{~nm}$, i.e., the gap size between gate $\mathrm{G}_{2}$ and $\mathrm{G}_{3}$. As a comparison, the dashed line shown in Fig. 4(b) represents the gate-voltage-tuned plasmon frequency with a cavity size of $150 \mathrm{~nm}$, i.e., the length of gate $\mathrm{G}_{1}$. This implies that the plasmons are excited and confined in the 2DEG enclosed by $\mathrm{G}_{2}$ and $\mathrm{G}_{3}$ instead of in the $2 \mathrm{DEG}$ right beneath the control gate $\mathrm{G}_{1}{ }^{5-7}$ 

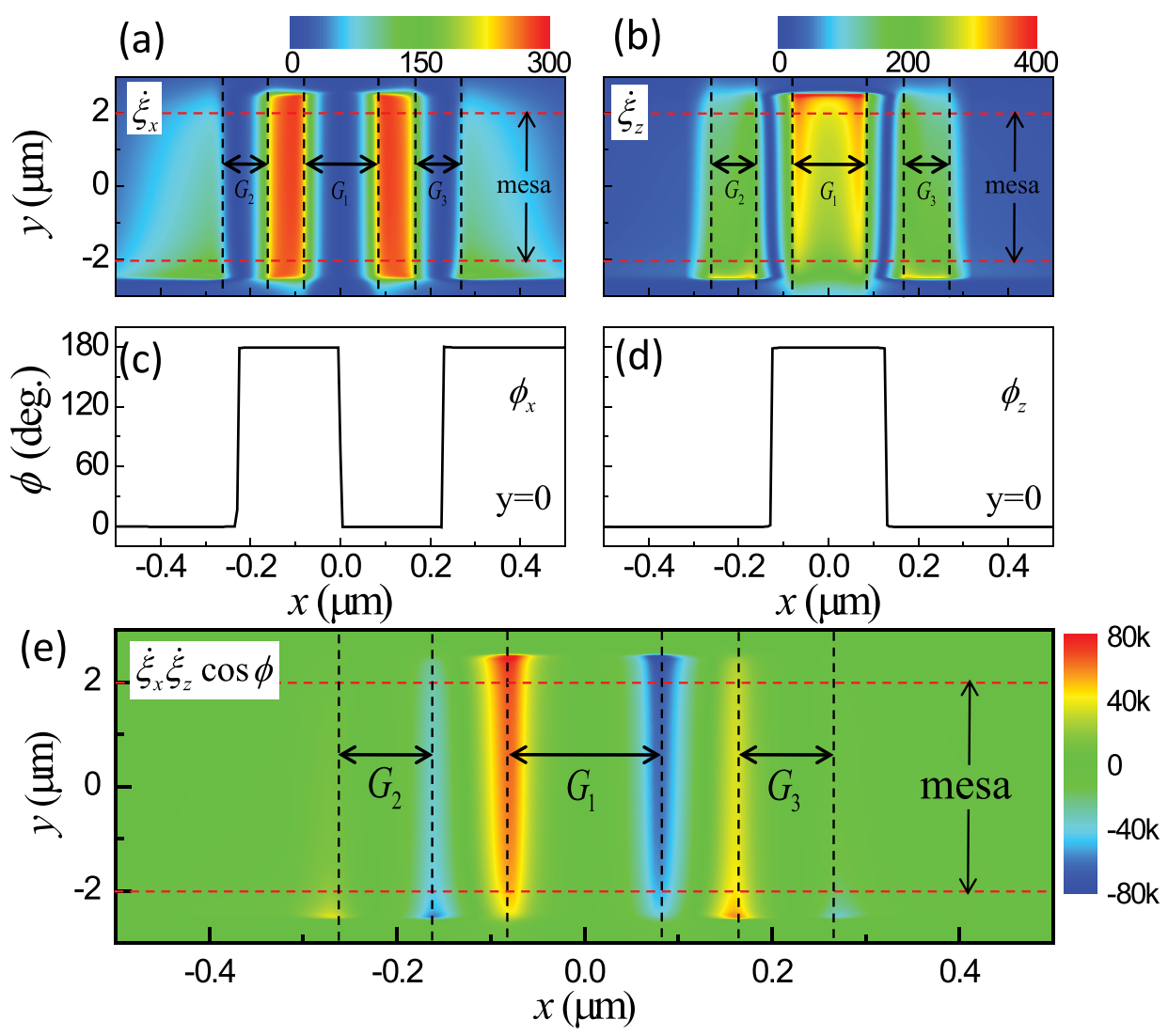

FIG. 2. Spatial distribution of the $\mathrm{THz}$ electric field and the phase from a FDTD simulation at $900 \mathrm{GHz}$. (a) Field enhancement factor $\dot{\xi}_{x}$. (b) Field enhancement factor $\dot{\xi}_{z}$. (c) and (d) The phases $\phi_{x}$ and $\phi_{z}$ at $y=0$. (e) Mixing factor $\dot{\xi}_{x} \dot{\xi}_{z} \cos \phi$.
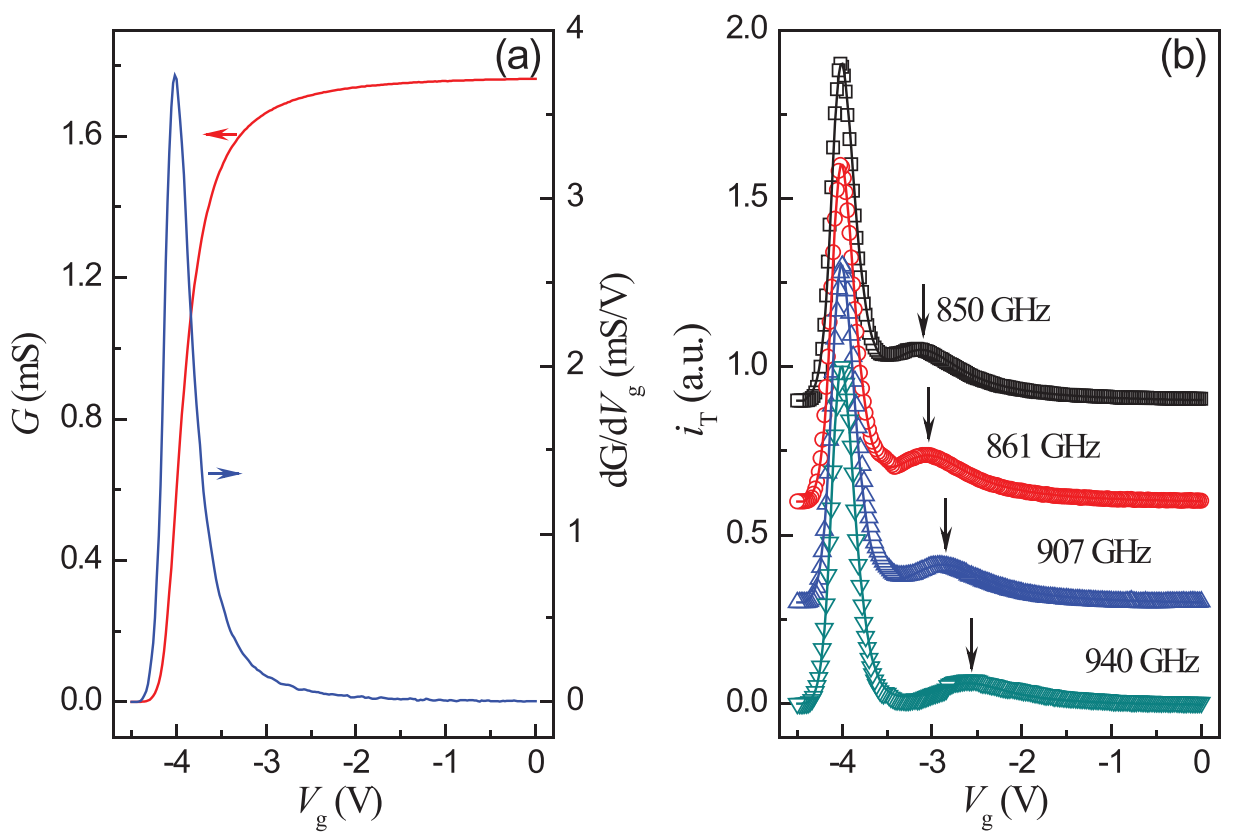

FIG. 3. (a) Channel conductance and its derivative as a function of the gate voltage. (b) THz response at different incident $\mathrm{THz}$ frequencies $(\square$ : 850 $\mathrm{GHz}, \bigcirc: 861 \mathrm{GHz}, \triangle: 907 \mathrm{GHz}$, and $\nabla: 940 \mathrm{GHz})$. The solid curves are calculated responses based on Eq. (4). The arrows mark the locations of plasmon resonances.

As can be seen in Fig. 3(b), the resonant response is much weaker than the nonresonant self-mixing response. The current responsivity is estimated to be $540 \mathrm{~mA} / \mathrm{W}$ and $59 \mathrm{~mA} / \mathrm{W}$ for the self-mixing response and the resonant response, respectively. The corresponding noise-equivalent powers are $3 \mathrm{pW} / \sqrt{\mathrm{Hz}}$ and $45 \mathrm{pW} / \sqrt{\mathrm{Hz}}$. Comparing to those detectors with a $2-\mu \mathrm{m}$ gate at room temperature reported previously, ${ }^{17}$ this nano-gated detector exhibits an unexpectedly low responsivity in the self-mixing mode at $77 \mathrm{~K}$. It is the symmetric antenna design that strongly suppresses the self-mixing response. The amplitude of the resonant response relies on how plasmons are effectively excited and how effectively the charge oscillations are converted into a direct current. The former can be characterized by the plasmon quality factor $Q=2 \pi f_{p} \tau_{p}$. The latter is a complex function of the boundary conditions of the plasmon cavity, and it is not yet specifically known. By substituting Eq. (2) into Eq. (1), we obtain the resonant response as a function of the plasmon frequency (Fig. 4(c)), and the plasmon quality factors can be extracted as shown in Fig. 4(d). We found a spectral resolution of better than $10 \mathrm{GHz}$ can be achieved by tuning the gate voltage. By fitting the 

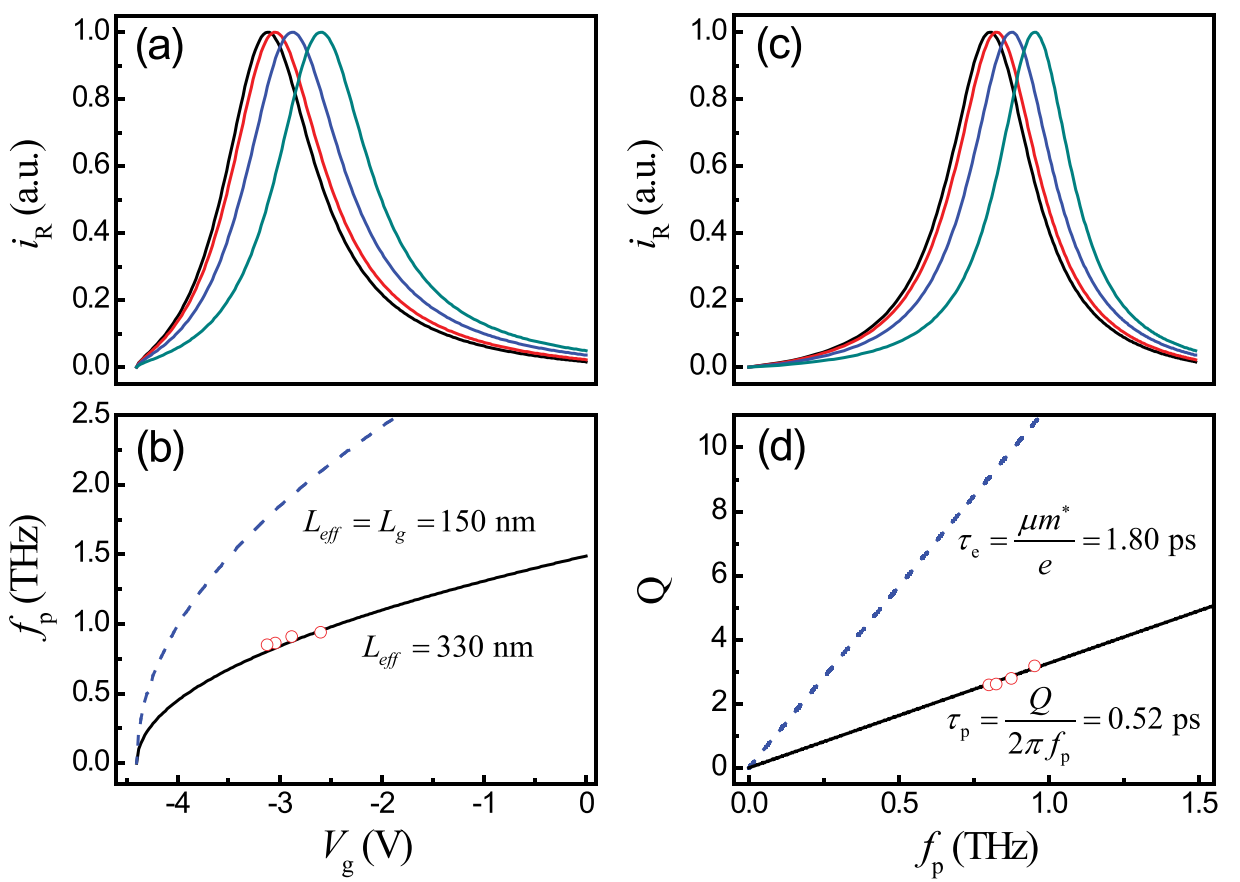

FIG. 4. (a) Resonant photocurrent as a function of the gate voltage. (b) Resonant plasmon frequency as a function of the gate voltage. The solid curve and the dashed curve correspond to an effective cavity length of $L_{\text {eff }}=330 \mathrm{~nm}$ and $L_{\text {eff }}=150 \mathrm{~nm}$, respectively. (c) Resonant photocurrent as a function of the plasmon frequency. (d) Quality factor of the plasmon resonance extracted from (c) as a function of the plasmon frequency. The solid curve and the dashed curve correspond to a plasmon relaxation time of $\tau_{p}=0.52 \mathrm{ps}$ and a transport relaxation time of $\tau_{e}=1.80 \mathrm{ps}$, respectively. experimentally extracted quality factors, we found that the effective plasmon lifetime is $\tau_{p}=0.52 \mathrm{ps}$, which is about one third of the electron's relaxation time $\tau_{e}=\mu m^{*} / e=1.80 \mathrm{ps}$ obtained from the Hall mobility at $77 \mathrm{~K}$. This indicates that damping of plasmon due to strong electron-electron and electron-plasmon interactions take place in the cavity. ${ }^{7}$

To further verify the symmetric design, we use the same technique as that reported in Ref. 18. The degree of symmetry in the photoresponse mapped in a $2 \mathrm{D}$ color-scale plot as a function of $V_{d s}$ and $V_{g}$ at $907 \mathrm{GHz}$ can be seen in Fig. 5(a). A strong self-mixing photocurrent is produced near $-4.00 \mathrm{~V}$ and is marked by the dashed line. When the large positive bias is applied at the drain (source) side, the 2DEG under the gate at the drain (source) side is depleted, and the locally induced negative (positive) photocurrent is suppressed effectively, as schematically shown in the insets of Fig. 5(b). Along the dashed line in Fig. 5(a), the measured
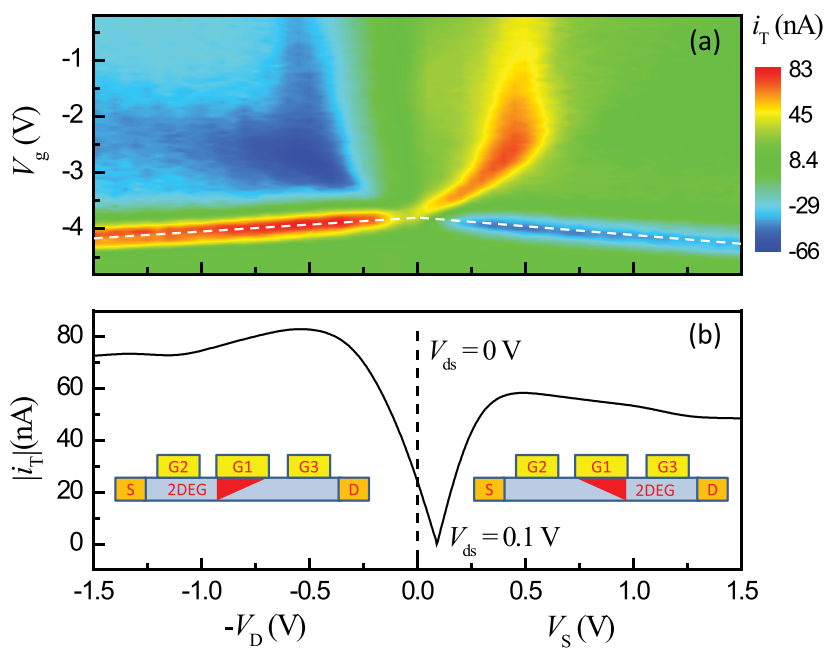

FIG. 5. (a) Examination of the degree of symmetry in the THz photocurrent mapped in a 2D color-scale plot as a function of $V_{d s}$ and $V_{g}$ at $907 \mathrm{GHz}$. (b) Absolute value of $\mathrm{THz}$ photocurrent as a function of $V_{d s}$ extracted along the dashed line in (a). photocurrent comes from the locally induced positive (negative) photocurrent at the source (drain) side of the gated 2DEG channel. The corresponding current responsivity increases from $0.54 \mathrm{~A} / \mathrm{W}$ to $1.72 \mathrm{~A} / \mathrm{W}$ as bias $V_{\mathrm{D}}$ changes from $0 \mathrm{~V}$ to $0.5 \mathrm{~V}$. As shown in Fig. 5(b), the absolute $\mathrm{THz}$ photocurrent as a function of $V_{d s}$ is extracted along the dashed line and is plotted in Fig. 5(a). The maximum photocurrent with the bias applied at the drain and at the source is $83 \mathrm{nA}$ and $57 \mathrm{nA}$, respectively. The expected location of the minimal photocurrent is shifted from $0 \mathrm{~V}$ to $V_{s}=0.1 \mathrm{~V}$. At $V_{d s}=0 \mathrm{~V}$, the photocurrent is about $26 \mathrm{nA}$, which is about the difference between the observed maximal responses when the bias is applied at the drain and at the source. This confirms that a residual asymmetry exists in our symmetrically designed device. It is this asymmetry that allows us to bring up the resonant response with a suppressed self-mixing photoresponse as the background. Since the broadband nonresonant response is always expected to occur, it is difficult to separate the resonant response from the nonresonant response when both the gate voltage and the source-drain bias are applied. However, in our symmetrically designed detector, we can set the detector at a proper operation point so that the residual asymmetry in selfmixing response can be electrically cancelled and minimize the self-mixing response, in our case $V_{s}=0.1 \mathrm{~V}$. On the other hand, by operating the detector with a large source-drain bias, the detector allows for $\mathrm{THz}$ detection with an enhanced sensitivity. Thus, a symmetrically designed detector could provide both high sensitivity and a spectral resolution.

In conclusion, we have demonstrated and analyzed both the resonant and nonresonant (self-mixing) $\mathrm{THz}$ photoresponses in a GaN/AlGaN 2DEG detector with symmetrically arranged nanogates, antennas, and filters. Experiments confirm that the self-mixing response can be effectively suppressed by the symmetric design. The observed self-mixing response at zero bias suggests that a certain degree of asymmetry exists in the $\mathrm{THz}$ field distribution and/or in the fieldeffect electron channel. The observed resonant response 
agrees well with the quarter-wavelength plasmon mode defined by the nanogates. Although this resonant photoresponse may be induced by the residual asymmetry in our symmetrically designed device, more indepth research is required for further engineering the boundary conditions for cavity plasmon modes. The broadband self-mixing mechanism and the narrowband resonant detection integrated in one detector may provide both high sensitiviy and spectral resolution.

The authors gratefully acknowledge the partial supports from the National Natural Science Foundation of China (Nos. 61401456, 61271157, and 61401297), the China Postdoctoral Science Foundation (2014M551678), the Jiangsu Planned Projects for Postdoctoral Research Funds (No. 1301054B), the Jiangsu Science Foundation Fund (No. BK20140283), and the Chinese Academy of Sciences visiting professorship for senior international scientists (No. 2010T2J07).

${ }^{1}$ M. Tonouchi, Nat. Photonics 1, 97 (2007).

${ }^{2}$ F. Sizova and A. Rogalski, Prog. Quantum Electron. 34, 278 (2010).

${ }^{3}$ M. I. Dyakonov and M. S. Shur, Phys. Rev. Lett. 71, 2465 (1993).

${ }^{4}$ M. I. Dyakonov and M. S. Shur, IEEE Trans. Electron Devices 43, 380 (1996).

${ }^{5}$ W. Knap, Y. Deng, S. Rumyantsev, J. Q. Lü, M. S. Shur, C. A. Saylor, and L. C. Brunel, Appl. Phys. Lett. 80, 3433 (2002).
${ }^{6}$ A. El Fatimy, F. Teppe, N. Dyakonova, W. Knap, A. Shchepetov, Y. Roelens, S. Bollaert, A. Cappy, and S. Rumyantsev, Appl. Phys. Lett. 89, 131926 (2006).

${ }^{7}$ V. V. Popov, O. V. Polischuk, W. Knap, and A. E. Fatimy, Appl. Phys. Lett. 93, 263503 (2008).

${ }^{8}$ S. Boubanga-Tombet, F. Teppe, D. Coquillat, S. Nadar, N. Dyakonova, H. Videlier, W. Knap, A. Shchepetov, C. Gardes, Y. Roelens, S. Bollaert, D. Seliuta, R. Vadoklis, and G. Valueis, Appl. Phys. Lett. 92, 212101 (2008).

${ }^{9}$ X. G. Peralta, S. J. Allen, M. C. Wanke, N. E. Harff, J. A. Simmons, M. P. Lilly, J. L. Reno, P. J. Burke, and J. P. Eisenstein, Appl. Phys. Lett. 81, 1627 (2002).

${ }^{10}$ G. C. Dyer, G. R. Aizin, S. J. Allen, A. D. Grine, D. Bethke, J. L. Reno, and E. A. Shaner, Nat. Photonics. 7, 925 (2013).

${ }^{11}$ T. Tanigawa, T. Onishi, S. Takigawa, and T. Otsuji, 68th Device Research Conference (DRC 2010) (IEEE, 2010), p. 167.

${ }^{12}$ S. Kim, J. D. Zimmerman, P. Focardi, A. C. Gossard, D. H. Wu, and M. S. Sherwin, Appl. Phys. Lett. 92, 253508 (2008).

${ }^{13}$ W. Knap, Y. Deng, S. Rumyantsev, and M. S. Shur, Appl. Phys. Lett. 81, 4637 (2002).

${ }^{14}$ T. Otsuji and M. Shur, IEEE Microwave Mag. 15, 43 (2014).

${ }^{15}$ A. Lisauskas, U. Pfeiffer, E. Öjefors, P. H. Bolivar, D. Glaab, and H. G. Roskos, J. Appl. Phys. 105, 114511 (2009).

${ }^{16}$ R. A. Hadi, H. Sherry, J. Grzyb, Y. Zhao, W. Förster, H. M. Keller, A. Cathelinand, A. Kaiser, and U. R. Pfeiffer, IEEE J. Solid-State Circuits 47, 2999 (2012).

${ }^{17}$ J. D. Sun, Y. F. Sun, H. Qin, B. S. Zhang, and D. M. Wu, Appl. Phys. Lett. 100, 013506 (2012).

${ }^{18}$ J. D. Sun, H. Qin, R. A. Lewis, Y. F. Sun, X. Y. Zhang, Y. Cai, D. M. Wu, and B. S. Zhang, Appl. Phys. Lett. 100, 173513 (2012).

${ }^{19}$ L. Vicarelli, M. S. Vitiello, D. Coquillat, A. Lombardo, A. C. Ferrari, W. Knap, M. Polini, V. Pellegrini, and A. Tredicucci, Nat. Mater. 11, 865 (2012).

${ }^{20}$ Y. F. Sun, J. D. Sun, Y. Zhou, R. B. Tan, C. H. Zeng, W. Xue, H. Qin, B. S. Zhang, and D. M. Wu, Appl. Phys. Lett. 98, 252103 (2011). 